\title{
On Bernstein-Schnabl Operators on the Unit Interval
}

\author{
Francesco Altomare, Vita Leonessa and Ioan Raşa \\ Dedicated to Professor József Szabados on the occasion of his 70th birthday
}

\begin{abstract}
In this paper we study the Bernstein-Schnabl operators associated with a continuous selection of Borel measures on the unit interval. We investigate their approximation properties by presenting several estimates of the rate of convergence in terms of suitable moduli of smoothness. We also study some shape preserving properties as well as the preservation of the convexity. Moreover we show that their iterates converge to a Markov semigroup whose generator is a degenerate second order elliptic differential operator on the unit interval. Qualitative properties of this semigroup are also investigated together with its asymptotic behaviour.
\end{abstract}

Keywords. Borel measure, positive approximation process, Bernstein-Schnabl operator, rate of convergence, asymptotic formula, degenerate differential operator, Markov semigroup, approximation of semigroups

Mathematics Subject Classification (2000). Primary 41A36, secondary 47D06, $35 \mathrm{~A} 35$

\section{Introduction}

Bernstein-Schnabl operators were first introduced by Schnabl (see [27, 28]) in the context of sets of probability Radon measures on compact Hausdorff spaces. Subsequently Grossman in [16] proposed a general method of constructing Bernstein-Schnabl operators on an arbitrary convex compact subset of a locally convex space and he showed that they are an approximation process for continuous functions. A particular class of these operators has been also studied by the first author [2] (see also [3]) and, subsequently, by several other authors (see [8, Chapter 6] and the relevant Notes and References). Their construction essentially involves positive projections [8, Section 6.1] and they satisfy many additional properties useful for the study of evolution problems [8, Section 6.2].

F. Altomare: Dipartimento di Matematica, Campus Universitario, Università di Bari, Italia; altomare@dm.uniba.it

V. Leonessa: Dipartimento di Matematica e Informatica, Università degli Studi della Basilicata, Potenza, Italia; vita.leonessa@unibas.it

I. Raşa: Departamento de Matematica, Univesitatea Technica of Cluj-Napoca, Romania; Ioan.Rasa@math.utcluj.ro 
In this paper we deepen the study of the Bernstein-Schnabl operators associated with a general continuous selection of probability Borel measures on the interval $[0,1]$, which does not necessarily arise from a positive projection. These operators seem to have some interest because they furnish new general approximation processes for continuous functions and they also approximate the solutions of the initial-boundary problems associated with a class of degenerate diffusion equations.

In the first section we recall their definition and discuss some examples of them. After that, we investigate their approximation properties and show several estimates of the rate of convergence by means of suitable moduli of smoothness. Shape preserving properties are discussed in Section 2. In particular, we investigate some conditions under which these operators preserve the convexity. In the third section we show that suitable iterates of BernsteinSchnabl operators converge to a Markov semigroup on $\mathcal{C}([0,1])$ whose generator is a degenerate differential operator of the form

$$
A u(x):=\alpha(x) u^{\prime \prime}(x) \quad(0<x<1)
$$

defined for every $u \in \mathcal{C}([0,1]) \cap \mathcal{C}^{2}(] 0,1[)$ satisfying $\lim _{x \rightarrow 0^{+}} \alpha(x) u^{\prime \prime}(x)=0=$ $\lim _{x \rightarrow 1^{-}} \alpha(x) u^{\prime \prime}(x)$ and other additional hypotheses. Here the function $\alpha$ is continuous and positive on $[0,1]$ and $0<\alpha(x) \leq \frac{x(1-x)}{2}(0<x<1)$. By means of Bernstein-Schnabl operators we establish some qualitative properties of this semigroup and, in particular, its asymptotic behaviour. In the same section we also study the generation properties of general differential operators of the form $(*)$ and determine suitable continuous selections of Borel measures such that the iterates of the corresponding Bernstein-Schnabl operators converge to the given Markov semigroup.

\section{Bernstein-Schnabl operators}

Throughout this paper we denote by $\mathcal{C}([0,1])$ the space of all real valued continuous functions on the interval $[0,1]$ endowed with the sup-norm $\|\cdot\|_{\infty}$.

Let $\mathcal{B}([0,1])$ be the $\sigma$-algebra of all Borel subsets of $[0,1]$ and denote by $M^{+}([0,1])$ the cone of all (regular) Borel measures on $[0,1]$. The support of any $\mu \in M^{+}([0,1])$ is denoted by $\operatorname{Supp} \mu$. For every $x \in[0,1]$ we denote by $\varepsilon_{x}$ the point-mass measure concentrated at $x$, i.e.,

$$
\varepsilon_{x}(B):=\left\{\begin{array}{ll}
1 & \text { if } x \in B, \\
0 & \text { if } x \notin B,
\end{array} \quad \text { for every } B \in \mathcal{B}([0,1])\right.
$$

The symbol 1 stands for the constant function 1 and, for every $n \geq 1$, $e_{n} \in \mathcal{C}([0,1])$ denotes the function $e_{n}(t):=t^{n}(0 \leq t \leq 1)$. 
A continuous selection of probability Borel measures on $[0,1]$ is a family $\left(\mu_{x}\right)_{0 \leq x \leq 1}$ of probability Borel measures on $[0,1]$ such that for every $f \in \mathcal{C}([0,1])$ the function

$$
x \longmapsto \int_{0}^{1} f d \mu_{x}
$$

is continuous on $[0,1]$. Such a function will be denoted by $T(f)$, i.e.,

$$
T(f)(x):=\int_{0}^{1} f d \mu_{x} \quad(0 \leq x \leq 1) .
$$

The operator $T: \mathcal{C}([0,1]) \longrightarrow \mathcal{C}([0,1])$ is positive (hence continuous) and $T(\mathbf{1})=\mathbf{1}($ and hence $\|T\|=1)$.

In fact, by Riesz representation theorem, each positive linear operator $T: \mathcal{C}([0,1]) \longrightarrow \mathcal{C}([0,1])$ such that $T(\mathbf{1})=\mathbf{1}$, generates a continuous selection of probability Borel measures on $[0,1]$ satisfying (1.2). Thus, all the definitions and results we shall develop in this paper, could be equally attributed to a general positive linear operator as above. However we prefer to handle Borel measures instead of linear operators for the sake of simplicity.

From now on we shall fix a continuous selection $\left(\mu_{x}\right)_{0 \leq x \leq 1}$ of probability Borel measures on $[0,1]$ satisfying the following additional assumption:

$$
\int_{0}^{1} e_{1} d \mu_{x}=x \quad(0 \leq x \leq 1)
$$

(i.e., $\left.T\left(e_{1}\right)=e_{1}\right)$. By Jensen's inequality [9, Theorem 3.9] it follows that $x^{2} \leq T\left(e_{2}\right)(x) \leq x(0 \leq x \leq 1)$. Therefore

$$
0 \leq T\left(e_{2}\right)(x)-x^{2} \leq x-x^{2}=x(1-x) \quad(0 \leq x \leq 1)
$$

By means of such a selection it is possible to define a sequence of positive linear operators.

Definition 1.1. For every $n \geq 1$, the $n$-th Bernstein-Schnabl operator associated with a given selection $\left(\mu_{x}\right)_{0 \leq x \leq 1}$ satisfying (1.3) is the positive linear operator $B_{n}: \mathcal{C}([0,1]) \longrightarrow \mathcal{C}([0,1])$ defined for every $f \in \mathcal{C}([0,1])$ and $x \in[0,1]$ as

$$
\begin{aligned}
B_{n}(f)(x): & =\int_{[0,1]^{n}} f\left(\frac{x_{1}+\cdots+x_{n}}{n}\right) d \mu_{x}^{n}\left(x_{1}, \ldots, x_{n}\right) \\
& =\int_{0}^{1} \cdots \int_{0}^{1} f\left(\frac{x_{1}+\cdots+x_{n}}{n}\right) d \mu_{x}\left(x_{1}\right) \cdots d \mu_{x}\left(x_{n}\right),
\end{aligned}
$$

where $\mu_{x}^{n}$ denotes the tensor product of $\mu_{x}$ with itself n-times. 
Each positive linear operator $B_{n}$ maps the space $\mathcal{C}([0,1])$ into itself because of (1.1), it is continuous with respect to the sup-norm and $\left\|B_{n}\right\|=1$ since $B_{n}(\mathbf{1})=\mathbf{1}$. Note that, if $\mu_{x}=x \varepsilon_{1}+(1-x) \varepsilon_{0}$ for all $x \in[0,1]$, they turn into the well-known Bernstein operators (see, e.g., [8, pp. 218-220]) which are defined by setting, for every $n \geq 1, f \in \mathcal{C}([0,1])$ and $x \in[0,1]$,

$$
\mathcal{B}_{n}(f)(x):=\sum_{k=0}^{n}\left(\begin{array}{l}
n \\
k
\end{array}\right) x^{k}(1-x)^{n-k} f\left(\frac{k}{n}\right) .
$$

Here we discuss some other examples.

\section{Examples 1.2.}

1. For every $x \in[0,1]$ consider the measure $\mu_{x}:=\alpha(x) \varepsilon_{0}+\beta(x) \varepsilon_{1 / 2}+\gamma(x) \varepsilon_{1}$, where $\alpha, \beta, \gamma \in C([0,1])$ are such that $0 \leq \alpha \leq 1,0 \leq \beta \leq 1,0 \leq \gamma \leq 1$, $\alpha+\beta+\gamma=1$ and $x=\frac{\beta(x)}{2}+\gamma(x)(0 \leq x \leq 1)$. The Bernstein-Schnabl operators are given by

$$
B_{n}(f)(x)=\sum_{h=0}^{n} \sum_{k=0}^{n-h}\left(\begin{array}{l}
n \\
h
\end{array}\right)\left(\begin{array}{c}
n-h \\
k
\end{array}\right) \alpha(x)^{n-h-k} \beta(x)^{h} \gamma(x)^{k} f\left(\frac{h+2 k}{2 n}\right)
$$

for every $f \in C([0,1]), x \in[0,1], n \geq 1$.

2. Let $\lambda \in C_{b}(] 0,1[)$ be a function satisfying $0 \leq \lambda \leq 1$ and, for every $0 \leq x \leq 1$, consider the probability Borel measure $\mu_{x}$ defined as

$$
\mu_{x}:= \begin{cases}\lambda(x)\left[x \varepsilon_{1}+(1-x) \varepsilon_{0}\right]+(1-\lambda(x)) \varepsilon_{x} & \text { if } 0<x<1 \\ \varepsilon_{x} & \text { if } x=0,1\end{cases}
$$

The family $\left(\mu_{x}\right)_{0 \leq x \leq 1}$ is a continuous selection of probability Borel measures satisfying (1.3) and the Bernstein-Schnabl operators associated with the continuous selection $\left(\mu_{x}\right)_{0 \leq x \leq 1}$ are the Lototsky-Schnabl operators defined by

$$
L_{n, \lambda}(f)(x)=\left\{\begin{aligned}
\sum_{h=0}^{n} \sum_{k=0}^{n-h}\left(\begin{array}{l}
n \\
h
\end{array}\right)\left(\begin{array}{c}
n-h \\
k
\end{array}\right) x^{k}(1-x)^{n-h-k} & \text { if } 0<x<1, \\
\times \lambda(x)^{n-h}(1-\lambda(x))^{h} f\left(\frac{k}{n}+\frac{h}{n} x\right) & \\
f(x) & \text { if } x=0,1,
\end{aligned}\right.
$$

for every $f \in C([0,1]), x \in[0,1], n \geq 1$ (see $[4,8])$.

The next identities turn out to be useful in studying the convergence of the Bernstein-Schnabl operators. They can be proved by direct calculations which 
we omit by the sake of brevity (see also [8, Section 6.1]). For every $n \geq 1$ the following identities hold true:

$$
\begin{aligned}
B_{n}(\mathbf{1}) & =\mathbf{1} \\
B_{n}\left(e_{1}\right) & =e_{1} \\
B_{n}\left(e_{2}\right) & =\frac{1}{n}\left[(n-1) e_{2}+T\left(e_{2}\right)\right], \\
B_{n}\left(e_{3}\right)= & \frac{1}{n^{2}}\left[T\left(e_{3}\right)+3(n-1) e_{1} T\left(e_{2}\right)+(n-1)(n-2) e_{3}\right], \\
B_{n}\left(e_{4}\right)= & \frac{1}{n^{3}}\left[T\left(e_{4}\right)+4(n-1) e_{1} T\left(e_{3}\right)+3(n-1) T\left(e_{2}\right)^{2}\right. \\
& \left.+6(n-1)(n-2) e_{2} T\left(e_{2}\right)+(n-1)(n-2)(n-3) e_{4}\right],
\end{aligned}
$$

where $T$ is given by (1.2).

Since the operators $B_{n}$ are linear and positive, by the Korovkin theorem (see, e.g., [8, Theorem 4.2.7]) and the above formulas it follows that $\left(B_{n}\right)_{n \geq 1}$ is a positive approximation process in the space $\mathcal{C}([0,1])$, i.e., for every $f \in \mathcal{C}([0,1])$

$$
\lim _{n \rightarrow \infty} B_{n}(f)=f \quad \text { uniformly on }[0,1]
$$

It is possible to estimate the rate of convergence described in (1.11). We shall actually present both pointwise and uniform estimates of the rate of convergence by means of the usual moduli of smoothness of first and second order $\omega(f, \delta)$ and $\omega_{2}(f, \delta)(\delta>0)$, respectively, defined as

$$
\omega(f, \delta):=\sup \{|f(x)-f(y)|:|x-y| \leq \delta, x, y \in[0,1]\}
$$

and

$$
\omega_{2}(f, \delta):=\sup \left\{\left|f(x)-2 f\left(\frac{x+y}{2}\right)+f(y)\right|:|x-y| \leq 2 \delta, x, y \in[0,1]\right\},
$$

for every real valued bounded function $f$ on $[0,1]$ and $\delta>0$ (see, e.g., $[8$, Section $5.1])$, as well as by the second order Ditzian-Totik modulus $\omega_{2}^{\varphi}(f, \delta)(\delta>0)$ defined by means of the weight function $\varphi(x):=\sqrt{x(1-x)}, x \in[0,1]$, as

$$
\omega_{2}^{\varphi}(f, \delta):=\sup \left\{\left|f(x)-2 f\left(\frac{x+y}{2}\right)+f(y)\right|:|x-y| \leq 2 \delta \varphi\left(\frac{x+y}{2}\right), x, y \in[0,1]\right\}
$$

for every real valued bounded function $f$ and $\delta>0$ on $[0,1]$ (see $[12,29]$ ).

We first need some preliminary remarks. As usual, for every $0 \leq x \leq 1$, we shall denote by $\psi_{x}$ the function $\psi_{x}(t):=t-x(0 \leq t \leq 1)$. It will be useful to evaluate, for every $n \in \mathbb{N}$ and $x \in[0,1]$, the moments of the first and second 
order of the operators $B_{n}$. Taking formulae (1.7) and (1.8) into account, for every $x \in[0,1]$, we get

$$
\begin{aligned}
& B_{n}\left(\psi_{x}\right)(x)=0 \\
& B_{n}\left(\psi_{x}^{2}\right)(x)=\frac{1}{n}\left(T\left(e_{2}\right)(x)-x^{2}\right) .
\end{aligned}
$$

If we set

$$
M:=\max _{0 \leq x \leq 1}\left(T\left(e_{2}\right)(x)-x^{2}\right),
$$

we further obtain $B_{n}\left(\psi_{x}^{2}\right)(x) \leq \frac{M}{n} \leq \frac{1}{4 n}$ (see (1.4)).

We are now in a position to show the following estimates, which would be compared with other ones stated for arbitrary convex compact subsets, e.g., in $[5]$ and $[20,21]$.

Theorem 1.3. Fix $n \geq 1, f \in \mathcal{C}([0,1])$ and $x \in[0,1]$. Then:

(1) $\left|B_{n}(f)(x)-f(x)\right| \leq\left(1+T\left(e_{2}\right)(x)-x^{2}\right) \omega\left(f, \frac{1}{\sqrt{n}}\right)$ and hence

$$
\left\|B_{n}(f)-f\right\|_{\infty} \leq(1+M) \omega\left(f, \frac{1}{\sqrt{n}}\right)
$$

where $M$ is given by (1.14);

(2) $\left|B_{n}(f)(x)-f(x)\right| \leq\left(1+\frac{T\left(e_{2}\right)(x)-x^{2}}{2}\right) \omega_{2}\left(f, \frac{1}{\sqrt{n}}\right)$ and hence

$$
\left\|B_{n}(f)-f\right\|_{\infty} \leq\left(1+\frac{M}{2}\right) \omega_{2}\left(f, \frac{1}{\sqrt{n}}\right)
$$

(3) $\left|B_{n}(f)(x)-f(x)\right| \leq \frac{3}{2}\left(1+\frac{T\left(e_{2}\right)(x)-x^{2}}{x(1-x)}\right) \omega_{2}^{\varphi}\left(f, \frac{1}{\sqrt{n}}\right)$

and hence

$$
\left\|B_{n}(f)-f\right\|_{\infty} \leq 3 \omega_{2}^{\varphi}\left(f, \frac{1}{\sqrt{n}}\right)
$$

Proof. By a general estimate proved in [8, Proposition 5.1.5], given $\delta>0$, by (1.8) we have

$$
\begin{aligned}
\left|B_{n}(f)(x)-f(x)\right| & \leq\left[1+\frac{1}{\delta^{2}}\left(B_{n}\left(e_{2}\right)(x)-e_{2}(x)\right)\right] \omega(f, \delta) \\
& =\left(1+\frac{1}{\delta^{2}} \frac{T\left(e_{2}\right)(x)-x^{2}}{n}\right) \omega(f, \delta) .
\end{aligned}
$$

Setting $\delta=\frac{1}{\sqrt{n}}$ we obtain the pointwise estimate of (1) and hence (1.15). 
By [22] (see also [15, Theorem 10]) we get

$$
\begin{aligned}
\left|B_{n}(f)(x)-f(x)\right| \leq & \left|B_{n}(\mathbf{1})(x)-1\right||f(x)|+\frac{1}{\delta}\left|B_{n}\left(\psi_{x}\right)(x)\right| \omega(f, \delta) \\
& +\left(B_{n}(\mathbf{1})(x)+\frac{1}{2 \delta^{2}} B_{n}\left(\psi_{x}^{2}\right)(x)\right) \omega_{2}(f, \delta) .
\end{aligned}
$$

Since $B_{n}(\mathbf{1})=1$ and $B_{n}\left(\psi_{x}\right)(x)=0$, from (1.19) it follows that

$$
\left|B_{n}(f)(x)-f(x)\right| \leq\left(1+\frac{1}{2 \delta^{2}} \frac{T\left(e_{2}\right)(x)-x^{2}}{n}\right) \omega_{2}(f, \delta) .
$$

Setting $\delta=\frac{1}{\sqrt{n}}$ we obtain the first estimate of (2) and hence (1.16).

We now observe that the Bernstein-Schnabl operators reproduce linear functions. By using a general estimate for positive linear operators reproducing linear functions [14, Theorem 15] we have

$$
\left|B_{n}(f)(x)-f(x)\right| \leq \frac{3}{2}\left(1+\frac{1}{\delta^{2}} \frac{B_{n}\left(\psi_{x}^{2}\right)(x)}{x(1-x)}\right) \omega_{2}^{\varphi}(f, \delta) .
$$

Replacing (1.13) in (1.20) and setting $\delta=\frac{1}{\sqrt{n}}$, we get (1.17). Finally (1.18) follows from (1.17) and (1.4).

\section{Some shape preserving properties of Bernstein-Schnabl operators}

In this section we prove that, under suitable hypotheses, Bernstein-Schnabl operators preserve the class of increasing continuous functions as well as the one of Hölder continuous functions. Finally we investigate the behaviour of the operators $B_{n}$ on convex functions.

Theorem 2.1. Consider the sequence of the operators $B_{n}$ defined by (1.5) and suppose that the operator $T$, given in (1.2), maps increasing functions into increasing functions. Then, for every $n \geq 1, B_{n}(f)$ is increasing on $[0,1]$, provided $f$ is increasing on $[0,1]$.

Proof. If $n=1$ the result is obvious because $B_{1}=T$. Suppose $n \geq 2$ and consider an increasing function $f \in \mathcal{C}([0,1])$ and $x \in[0,1]$. We shall use some auxiliary functions introduced in [26] (see also [8, Theorem 6.1.21]).

For every $x_{1}, \ldots, x_{n-1} \in[0,1]$ we consider the function $f_{x_{1}, \ldots, x_{n-1}}^{x}:[0,1] \longrightarrow \mathbb{R}$ defined by

$$
f_{x_{1}, \ldots, x_{n-1}}^{x}(t):=f\left(\frac{x_{1}+\cdots+x_{n-1}+t}{n}\right) \quad(0 \leq t \leq 1) .
$$


Moreover, for every $k=2, \ldots, n-1$ we consider the functions on $[0,1]$ defined by the following recursive formula:

$$
f_{x_{1}, \ldots, x_{n-k}}^{x}(t):=T\left(f_{x_{1}, \ldots, x_{n-k, t}}^{x}\right)(x) \quad(0 \leq t \leq 1) .
$$

Finally we define

$$
f^{x}(t):=T\left(f_{t}^{x}\right)(x) \quad(0 \leq t \leq 1) .
$$

By finite induction it is easy to prove that $f_{x_{1}, \cdots, x_{n-k}}^{x}$ is increasing for every $k=1, \ldots, n-1$. Moreover, we observe that $f^{x}$ is increasing as well.

Now let $y \in[0,1], x<y$ and observe that for every $k=1, \ldots, n-1$

$$
\begin{gathered}
T\left(f_{x_{1}, \ldots, x_{k}}^{y}\right)(x) \leq T\left(f_{x_{1}, \ldots, x_{k}}^{y}\right)(y) \\
T\left(f^{y}\right)(x) \leq T\left(f^{y}\right)(y)
\end{gathered}
$$

Since $f_{x_{1}, \ldots, x_{n-1}}^{x}=f_{x_{1}, \ldots, x_{n-1}}^{y}$, we have

$$
\begin{aligned}
B_{n}(f)(x) & =\int_{[0,1]^{n}} f\left(\frac{x_{1}+\cdots+x_{n}}{n}\right) d \mu_{x}^{n}\left(x_{1}, \ldots, x_{n}\right) \\
& =\int_{[0,1]^{n-1}} \int_{0}^{1} f_{x_{1}, \ldots, x_{n-1}}^{x}\left(x_{n}\right) d \mu_{x}\left(x_{n}\right) d \mu_{x}^{n-1}\left(x_{1}, \ldots, x_{n-1}\right) \\
& =\int_{[0,1]^{n-1}} T\left(f_{x_{1}, \ldots, x_{n-1}}^{x}\right)(x) d \mu_{x}^{n-1}\left(x_{1}, \ldots, x_{n-1}\right) \\
& =\int_{[0,1]^{n-1}} T\left(f_{x_{1}, \ldots, x_{n-1}}^{y}\right)(x) d \mu_{x}^{n-1}\left(x_{1}, \ldots, x_{n-1}\right) .
\end{aligned}
$$

From (2.2) it follows that

$$
\begin{aligned}
B_{n}(f)(x) & \leq \int_{[0,1]^{n-1}} T\left(f_{x_{1}, \ldots, x_{n-1}}^{y}\right)(y) d \mu_{x}^{n-1}\left(x_{1}, \ldots, x_{n-1}\right) \\
& =\int_{[0,1]^{n-2}}\left[\int_{0}^{1} f_{x_{1}, \ldots, x_{n-2}}^{y}\left(x_{n-1}\right) d \mu_{x}\left(x_{n-1}\right)\right] d \mu_{x}^{n-2}\left(x_{1}, \ldots, x_{n-2}\right) \\
& =\int_{[0,1]^{n-2}} T\left(f_{x_{1}, \ldots, x_{n-2}}^{y}\right)(x) d \mu_{x}^{n-2}\left(x_{1}, \ldots, x_{n-2}\right) \\
& \leq \int_{[0,1]^{n-2}} T\left(f_{x_{1}, \ldots, x_{n-2}}^{y}\right)(y) d \mu_{x}^{n-2}\left(x_{1}, \ldots, x_{n-2}\right) \\
& =\int_{[0,1]^{n-2}} f_{x_{1}, \ldots, x_{n-3}}^{y}\left(x_{n-2}\right) d \mu_{x}^{n-2}\left(x_{1}, \ldots, x_{n-2}\right) \\
& \leq \cdots \leq \int_{0}^{1} T\left(f_{x_{1}}^{y}\right)(x) d \mu_{x}\left(x_{1}\right)
\end{aligned}
$$


and, by (2.3),

$$
B_{n}(f)(x) \leq \int_{0}^{1} T\left(f_{x_{1}}^{y}\right)(y) d \mu_{x}\left(x_{1}\right)=\int_{0}^{1} f^{y}\left(x_{1}\right) d \mu_{x}\left(x_{1}\right)=T\left(f^{y}\right)(x) \leq T\left(f^{y}\right)(y) .
$$

On the other hand $B_{n}(f)(y)=T\left(f^{y}\right)(y)$, because

$$
\begin{aligned}
B_{n}(f)(y) & =\int_{[0,1]^{n}} f\left(\frac{x_{1}+\cdots+x_{n}}{n}\right) d \mu_{y}^{n}\left(x_{1}, \ldots, x_{n}\right) \\
& =\int_{[0,1]^{n-1}} \int_{0}^{1} f_{x_{1}, \ldots, x_{n-1}}^{y}\left(x_{n}\right) d \mu_{y}\left(x_{n}\right) d \mu_{y}^{n-1}\left(x_{1}, \ldots, x_{n-1}\right) \\
& =\int_{[0,1]^{n-1}} T\left(f_{x_{1}, \ldots, x_{n-1}}^{y}\right)(y) d \mu_{y}^{n-1}\left(x_{1}, \ldots, x_{n-1}\right) \\
& =\int_{[0,1]^{n-1}} f_{x_{1}, \ldots, x_{n-2}}^{y}\left(x_{n-1}\right) d \mu_{y}\left(x_{n-1}\right) d \mu_{y}^{n-2}\left(x_{1}, \ldots, x_{n-2}\right) \\
& =\int_{[0,1]^{n-2}} T\left(f_{x_{1}, \ldots, x_{n-2}}^{y}\right)(y) d \mu_{y}^{n-2}\left(x_{1}, \ldots, x_{n-2}\right) \\
& =\cdots=\int_{0}^{1} T\left(f_{x_{1}}^{y}\right)(y) d \mu_{y}\left(x_{1}\right)=T\left(f^{y}\right)(y) .
\end{aligned}
$$

Accordingly, since $x, y \in[0,1]$ were arbitrarily chosen, the proof is complete.

Remark 2.2. In the particular case of Examples 1.2, 2, with $\lambda$ constant, the above result has been proved in [1, Theorem 1].

Now we proceed to show the property of preserving classes of Hölder continuous functions. For given $M>0$ and $0 \leq \alpha \leq 1$ we shall denote by $\operatorname{Lip}_{M} \alpha$ the set of all $f \in \mathcal{C}([0,1])$ such that $|f(x)-f(y)| \leq M|x-y|^{\alpha}$ for every $x, y \in[0,1]$.

We have the following result whose proof follows the same line of [26] (see also [8, Theorem 6.1.21]) and so we omit it.

Theorem 2.3. Let $\left(B_{n}\right)_{n \geq 1}$ be the sequence of the Bernstein-Schnabl operators defined by (1.5) and suppose that there exists $c \geq 1$ such that

$$
T(f) \in \operatorname{Lip}_{c} 1 \quad \text { for every } f \in \operatorname{Lip}_{1} 1 \text {, }
$$

where $T$ is given by (1.2). Then, $B_{n}(f) \in \operatorname{Lip}_{c M} 1$ for every $f \in \operatorname{Lip}_{M} 1$ and $n \geq 1$.

Since $\left\|B_{n}\right\|=1$, according to the above theorem together with Corollary 6.1.20 in [8], we immediately have the further corollary. 
Corollary 2.4. If $T\left(\right.$ Lip $\left._{1} 1\right) \subset$ Lip $_{c} 1$ for some $c \geq 1$, then for every $n \geq 1$, $f \in \mathcal{C}([0,1]), \delta>0, M>0$ and $0<\alpha \leq 1$

$$
\omega\left(B_{n}(f), \delta\right) \leq(1+c) \omega(f, \delta) \quad \text { and } \quad B_{n}\left(\operatorname{Lip}_{M} \alpha\right) \subset \operatorname{Lip}_{c^{\alpha} M} \alpha .
$$

In particular, if $T\left(\operatorname{Lip}_{1} 1\right) \subset \operatorname{Lip}_{1} 1$, then

$$
\omega\left(B_{n}(f), \delta\right) \leq 2 \omega(f, \delta) \quad \text { and } \quad B_{n}\left(\operatorname{Lip}_{M} \alpha\right) \subset \operatorname{Lip}_{M} \alpha .
$$

As regards the behaviour of Bernstein-Schnabl operators on convex functions we already know from general results for convex compact sets that, if $f \in \mathcal{C}([0,1])$ is convex, then

$$
f \leq B_{n+1}(f) \leq B_{n}(f) \leq T(f)
$$

(see $[8,24,25])$.

Next we investigate some conditions under which the Bernstein-Schnabl operators preserve the convexity. This is certainly true for the classical Bernstein operators and in the case of the Lototsky-Schnabl operators with $\lambda$ constant in $[0,1]$ (see $[1$, Theorem 1] and [8, Theorem 6.1.21]). For the general case we shall require additional hypotheses on the continuous selection $\left(\mu_{x}\right)_{0 \leq x \leq 1}$. More precisely we assume that:

$\left(c_{1}\right)$ The operator $T$, given in (1.2), maps continuous convex functions into (continuous) convex functions;

$\left(c_{2}\right)$ For every convex function $f \in \mathcal{C}([0,1])$ and for every $x, y \in[0,1]$

$$
\int_{[0,1]^{2}} \varphi_{f} d\left(\mu_{x} \otimes \mu_{x}+\mu_{y} \otimes \mu_{y}\right) \geq 2 \int_{[0,1]^{2}} \varphi_{f} d\left(\mu_{x} \otimes \mu_{y}\right)
$$

where $\varphi_{f}(s, t):=f\left(\frac{s+t}{2}\right),(s, t) \in[0,1]^{2}$ and the symbol $\otimes$ denotes the tensor product of measures.

For a given $n \geq 1$ and $f \in \mathcal{C}([0,1])$ set

$$
F_{n}\left(f ; x_{1}, \ldots, x_{n}\right):=\int_{0}^{1} \ldots \int_{0}^{1} f\left(\frac{t_{1}+\cdots+t_{n}}{n}\right) d \mu_{x_{1}}\left(t_{1}\right) \ldots d \mu_{x_{n}}\left(t_{n}\right),
$$

where $x_{i} \in[0,1]$, for every $i=1, \ldots, n$.

We first observe that the function $F_{n}(f ; \ldots)$ is invariant with respect to any permutation of the indices $1, \ldots, n . F_{n}(f ; \ldots)$ is convex with respect to each variable $x_{i}, i=1, \ldots, n$. Finally, for all $x_{i} \in[0,1], i=1, \ldots, n$, by using $(2.5)$ we can write

$$
\begin{aligned}
& 2 F_{n}\left(f ; x_{1}, \ldots, x_{i}, x_{i+1}, \ldots, x_{n}\right) \\
& \quad \leq F_{n}\left(f ; x_{1}, \ldots, x_{i}, x_{i}, \ldots, x_{n}\right)+F_{n}\left(f ; x_{1}, \ldots, x_{i+1}, x_{i+1}, \ldots, x_{n}\right) .
\end{aligned}
$$


Moreover, for every $p, q \geq 0, p+q=n$, and for every $x, y \in[0,1]$ set

$$
S_{n, p, q}(f ; x, y):=F_{n}(f ; \underbrace{x, \ldots, x}_{p}, \underbrace{y, \ldots, y}_{q})+F_{n}(f ; \underbrace{x, \ldots, x}_{q}, \underbrace{y, \ldots, y}_{p}) .
$$

Note that

$$
S_{n, p, q}(f ; x, y)=S_{n, q, p}(f ; x, y)
$$

and the following lemma holds.

Lemma 2.5. Under hypotheses $\left(c_{1}\right)$ and $\left(c_{2}\right)$, for every convex $f \in \mathcal{C}([0,1])$, $k \geq 1$ and $x, y \in[0,1]$ we have

$$
S_{k, k-1,1}(f ; x, y) \leq S_{k, k, 0}(f ; x, y) .
$$

Proof. For $k=1,(2.9)$ is satisfied by virtue of (2.8). Assume that $k=2 m+1$ with $m \geq 1$. By (2.6) and (2.7) we obtain

$$
\begin{aligned}
2 S_{k, 2 m, 1}(f ; x, y)= & 2 F_{k}(f ; x, \ldots, x, y)+2 F_{k}(f ; x, y, \ldots, y) \\
\leq & F_{k}(f ; x, \ldots, x, x, x)+F_{k}(f ; x, \ldots, x, y, y) \\
& +F_{k}(f ; x, x, y, \ldots, y)+F_{k}(f ; y, y, y, \ldots, y) \\
= & S_{k, 2 m+1,0}(f ; x, y)+S_{k, 2 m-1,2}(f ; x, y) .
\end{aligned}
$$

Hence $S_{k, 2 m, 1}(f ; x, y) \leq S_{k, 2 m+1,0}(f ; x, y)+S_{k, 2 m-1,2}(f ; x, y)$. With an analogue reasoning it is possible to prove the following inequalities:

$$
\begin{aligned}
2 S_{k, 2 m-1,2}(f ; x, y) & \leq S_{k, 2 m, 1}(f ; x, y)+S_{k, 2 m-2,3}(f ; x, y) \\
2 S_{k, 2 m-2,3}(f ; x, y) & \leq S_{k, 2 m-1,2}(f ; x, y)+S_{k, 2 m-3,4}(f ; x, y) \\
& \vdots \\
2 S_{k, m+1, m}(f ; x, y) & \leq S_{k, m+2, m-1}(f ; x, y)+S_{k, m, m+1}(f ; x, y) .
\end{aligned}
$$

From (2.8) it follows that $S_{k, m+1, m}(f ; x, y)=S_{k, m, m+1}(f ; x, y)$ and so

$$
S_{k, m+1, m}(f ; x, y) \leq S_{k, m+2, m-1}(f ; x, y) .
$$

Subsequently we have $S_{k, m+1, m}(f ; x, y) \leq S_{k, m+2, m-1}(f ; x, y) \leq S_{k, m+3, m-2}(f ; x, y)$ $\leq \cdots \leq S_{k, 2 m+1,0}(f ; x, y)$ and the last inequality actually gives (2.9). Assume now that $k=2 m, m \geq 1$. With an analogue reasoning we get

$$
\begin{aligned}
2 S_{k, 2 m-1,1}(f ; x, y) & \leq S_{k, 2 m, 0}(f ; x, y)+S_{k, 2 m-2,2}(f ; x, y), \\
2 S_{k, 2 m-2,2}(f ; x, y) & \leq S_{k, 2 m-1,1}(f ; x, y)+S_{k, 2 m-3,3}(f ; x, y), \\
& \vdots \\
2 S_{k, m, m}(f ; x, y) & \leq S_{k, m+1, m-1}(f ; x, y)+S_{k, m-1, m+1}(f ; x, y) .
\end{aligned}
$$


Since $S_{k, m+1, m-1}(f ; x, y)=S_{k, m-1, m+1}(f ; x, y)$ we have

$$
S_{k, m, m}(f ; x, y) \leq S_{k, m+1, m-1}(f ; x, y)
$$

and hence $S_{k, m, m}(f ; x, y) \leq S_{k, m+1, m-1}(f ; x, y) \leq \cdots \leq S_{k, 2 m, 0}(f ; x, y)$. And, again, the last inequality gives (2.9).

At this point we can state the following result.

Theorem 2.6. Consider the Bernstein-Schnabl operators $B_{n}$ associated with a continuous selection of probability Borel measures $\left(\mu_{x}\right)_{0 \leq x \leq 1}$ defined by (1.5) and assume that $\left(c_{1}\right)$ and $\left(c_{2}\right)$ hold true. Then, for every $n \geq 1$ and for every convex function $f \in \mathcal{C}([0,1]), B_{n}(f)$ is convex on $[0,1]$.

Proof. If $n=1, B_{1}(f)=T(f)$ is convex. Suppose $n \geq 2$ and consider a convex function $f \in \mathcal{C}([0,1])$. Since $B_{n}(f)$ is continuous, it is sufficient to show that, for every $x, y \in[0,1]$,

$$
B_{n}(f)\left(\frac{x+y}{2}\right) \leq \frac{1}{2} B_{n}(f)(x)+\frac{1}{2} B_{n}(f)(y) .
$$

For every $x_{1}, \ldots, x_{n-1} \in[0,1]$ consider the auxiliary function defined by (2.1). Condition $\left(c_{1}\right)$ implies that the function $x \in[0,1] \longmapsto T\left(f_{x_{1}, \ldots, x_{n-1}}^{x}\right)(x)$ is convex too, because $f_{x_{1}, \ldots, x_{n-1}}^{x}$ is convex. Moreover

$$
T\left(f_{x_{1}, \ldots, x_{n-1}}^{\frac{x+y}{2}}\right)\left(\frac{x+y}{2}\right) \leq \frac{1}{2} T\left(f_{x_{1}, \ldots, x_{n-1}}^{x}\right)(x)+\frac{1}{2} T\left(f_{x_{1}, \ldots, x_{n-1}}^{y}\right)(y)
$$

for every $x, y \in[0,1]$, since $f_{x_{1}, \ldots, x_{n-1}}^{x}=f_{x_{1}, \ldots, x_{n-1}}^{y}$.

We now proceed by reasoning by induction on $n$. Suppose that $(2.10)$ is true for $n-1$ and consider the function $\delta_{n}\left(f, x_{n}\right)$ defined by

$$
\delta_{n}\left(f, x_{n}\right)(t):=f\left(\frac{n-1}{n} t+\frac{1}{n} x_{n}\right) \quad(0 \leq t \leq 1) .
$$

Then

$$
\begin{aligned}
B_{n}(f)\left(\frac{x+y}{2}\right) & =\int_{[0,1]^{n}} f\left(\frac{x_{1}+\cdots+x_{n}}{n}\right) d \mu_{\frac{x+y}{2}}^{n}\left(x_{1}, \ldots, x_{n}\right) \\
& =\int_{[0,1]^{n}} \delta_{n}\left(f, x_{n}\right)\left(\frac{x_{1}+\cdots+x_{n-1}}{n-1}\right) d \mu_{\frac{x+y}{2}}^{n}\left(x_{1}, \ldots, x_{n}\right) \\
& =\int_{0}^{1} B_{n-1}\left(\delta_{n}\left(f, x_{n}\right)\right)\left(\frac{x+y}{2}\right) d \mu_{\frac{x+y}{2}}\left(x_{n}\right) .
\end{aligned}
$$


Since (2.10) is true for $n-1$, we get

$$
\begin{aligned}
B_{n}(f)\left(\frac{x+y}{2}\right) \leq & \frac{1}{2} \int_{0}^{1} B_{n-1}\left(\delta_{n}\left(f, x_{n}\right)\right)(x) d \mu_{\frac{x+y}{2}}\left(x_{n}\right) \\
& +\frac{1}{2} \int_{0}^{1} B_{n-1}\left(\delta_{n}\left(f, x_{n}\right)\right)(y) d \mu_{\frac{x+y}{2}}\left(x_{n}\right)
\end{aligned}
$$

and, by the definitions of $B_{n-1}$ and $\delta_{n}\left(f, x_{n}\right)$, we obtain

$$
\begin{aligned}
B_{n}(f)\left(\frac{x+y}{2}\right) \leq & \frac{1}{2} \int_{[0,1]^{n-1}} \int_{0}^{1} f\left(\frac{x_{1}+\cdots+x_{n}}{n}\right) d \mu_{\frac{x+y}{2}}\left(x_{n}\right) d \mu_{x}^{n-1}\left(x_{1}, \ldots, x_{n-1}\right) \\
& +\frac{1}{2} \int_{[0,1]^{n-1}} \int_{0}^{1} f\left(\frac{x_{1}+\cdots+x_{n}}{n}\right) d \mu_{\frac{x+y}{2}}\left(x_{n}\right) d \mu_{y}^{n-1}\left(x_{1}, \ldots, x_{n-1}\right) \\
= & \frac{1}{2} \int_{[0,1]^{n-1}} T\left(f_{x_{1}, \ldots, x_{n-1}}^{\frac{x+y}{2}}\right)\left(\frac{x+y}{2}\right) d \mu_{x}^{n-1}\left(x_{1}, \ldots, x_{n-1}\right) \\
& +\frac{1}{2} \int_{[0,1]^{n-1}} T\left(f_{x_{1}, \ldots, x_{n-1}}^{\frac{x+y}{2}}\right)\left(\frac{x+y}{2}\right) d \mu_{y}^{n-1}\left(x_{1}, \ldots, x_{n-1}\right) .
\end{aligned}
$$

Now, by (2.11) we have

$$
\begin{aligned}
B_{n}(f)\left(\frac{x+y}{2}\right) \leq & \frac{1}{4} \int_{[0,1]^{n-1}} T\left(f_{x_{1}, \ldots, x_{n-1}}^{x}\right)(x) d \mu_{x}^{n-1}\left(x_{1}, \ldots, x_{n-1}\right) \\
& +\frac{1}{4} \int_{[0,1]^{n-1}} T\left(f_{x_{1}, \ldots, x_{n-1}}^{y}\right)(y) d \mu_{x}^{n-1}\left(x_{1}, \ldots, x_{n-1}\right) \\
& +\frac{1}{4} \int_{[0,1]^{n-1}} T\left(f_{x_{1}, \ldots, x_{n-1}}^{x}\right)(x) d \mu_{y}^{n-1}\left(x_{1}, \ldots, x_{n-1}\right) \\
& +\frac{1}{4} \int_{[0,1]^{n-1}} T\left(f_{x_{1}, \ldots, x_{n-1}}^{y}\right)(y) d \mu_{y}^{n-1}\left(x_{1}, \ldots, x_{n-1}\right) \\
= & \frac{1}{4} \int_{[0,1]^{n-1}} \int_{0}^{1} f_{x_{1}, \ldots, x_{n-1}}^{x}\left(x_{n}\right) d \mu_{x}\left(x_{n}\right) d \mu_{x}^{n-1}\left(x_{1}, \ldots, x_{n-1}\right) \\
& +\frac{1}{4} \int_{[0,1]^{n-1}} \int_{0}^{1} f_{x_{1}, \ldots, x_{n-1}}^{y}\left(x_{n}\right) d \mu_{y}\left(x_{n}\right)(y) d \mu_{x}^{n-1}\left(x_{1}, \ldots, x_{n-1}\right) \\
& +\frac{1}{4} \int_{[0,1]^{n-1}} \int_{0}^{1} f_{x_{1}, \ldots, x_{n-1}}^{x}\left(x_{n}\right) d \mu_{x}\left(x_{n}\right) d \mu_{y}^{n-1}\left(x_{1}, \ldots, x_{n-1}\right) \\
& +\frac{1}{4} \int_{[0,1]^{n-1}}^{1} \int_{0}^{1} f_{x_{1}, \ldots, x_{n-1}}^{y}\left(x_{n}\right) d \mu_{y}\left(x_{n}\right) d \mu_{y}^{n-1}\left(x_{1}, \ldots, x_{n-1}\right),
\end{aligned}
$$


that is

$$
\begin{aligned}
B_{n}(f)\left(\frac{x+y}{2}\right) \leq & \frac{1}{4} B_{n}(f)(x)+\frac{1}{4} B_{n}(f)(y) \\
& +\frac{1}{4} \int_{0}^{1} \int_{[0,1]^{n-1}} f\left(\frac{x_{1}+\cdots+x_{n}}{n}\right) d \mu_{x}^{n-1}\left(x_{1}, \ldots, x_{n-1}\right) d \mu_{y}\left(x_{n}\right) \\
& +\frac{1}{4} \int_{0}^{1} \int_{[0,1]^{n-1}} f\left(\frac{x_{1}+\cdots+x_{n}}{n}\right) d \mu_{y}^{n-1}\left(x_{1}, \ldots, x_{n-1}\right) d \mu_{x}\left(x_{n}\right) .
\end{aligned}
$$

Note that $B_{n}(f)(x)=F_{n}(f ; x, \ldots, x)$ and hence the last inequality turns into

$$
\begin{aligned}
B_{n}(f) & \left(\frac{x+y}{2}\right) \\
& \leq \frac{1}{4} B_{n}(f)(x)+\frac{1}{4} F_{n}(f ; x, \ldots, x, y)+\frac{1}{4} F_{n}(f ; y, \ldots, y, x)+\frac{1}{4} B_{n}(f)(y) \\
& =\frac{1}{4} B_{n}(f)(x)+\frac{1}{2} S_{n, n-1,1}(f ; x, y)+\frac{1}{4} B_{n}(f)(y) .
\end{aligned}
$$

Finally, by (2.9) for $k=n$ and by (2.7) and (2.8),

$$
\begin{aligned}
B_{n}(f)\left(\frac{x+y}{2}\right) & \leq \frac{1}{4} B_{n}(f)(x)+\frac{1}{2} S_{n, n, 0}(f ; x, y)+\frac{1}{4} B_{n}(f)(y) \\
& =\frac{1}{2} B_{n}(f)(x)+\frac{1}{2} B_{n}(f)(y),
\end{aligned}
$$

since

$$
2 S_{n, n, 0}(f ; x, y)=F_{n}(f ; x, \ldots, x)+F_{n}(f ; y, \ldots, y)=B_{n}(f)(x)+B_{n}(f)(y) .
$$

This completes the proof.

Examples 2.7. Examples of measures satisfying the hypotheses $\left(c_{1}\right)$ and $\left(c_{2}\right)$ of the previous theorem are illustrated below:

1. $\mu_{x}:=(1-x) \varepsilon_{0}+x \varepsilon_{1}(0 \leq x \leq 1)$.

2. For a given $\lambda \in[0,1]$, set $\mu_{x}:=(1-\lambda) \varepsilon_{x}+\lambda(1-x) \varepsilon_{0}+\lambda x \varepsilon_{1}(0 \leq x \leq 1)$.

3. Consider a concave function $\beta \in \mathcal{C}([0,1])$ such that $0 \leq \beta(x) \leq \min \{x, 1-x\}$ and set $\mu_{x}:=(1-x-\beta(x)) \varepsilon_{0}+2 \beta(x) \varepsilon_{1 / 2}+(x-\beta(x)) \varepsilon_{1}$.

\section{Bernstein-Schnabl operators and their associated Markov semigroups}

In this last section we show that suitable iterates of Bernstein-Schnabl operators converge to a Markov semigroup on $\mathcal{C}([0,1])$ whose generator is a one-dimensional second-order elliptic degenerate differential operator. We also study some 
qualitative properties of this semigroup and, in particular, its asymptotic behaviour. In the last part of the section we determine those differential operators which generate those Markov semigroups which can be represented by iterates of suitable Bernstein-Schnabl operators.

In the sequel we denote by $\mathcal{C}_{0}(] 0,1[)$ the space of all continuous functions on ] $0,1\left[\right.$ which vanish at 0 and 1 . This space is a closed subspace of $\mathcal{C}_{b}(] 0,1[)$.

Let $\left(\mu_{x}\right)_{0 \leq x \leq 1}$ be a continuous selection of probability Borel measures on $[0,1]$ such that $\int_{0}^{1} e_{1} d \mu_{x}=x$ for every $x \in[0,1]$.

In Section 1 we have already observed that $0 \leq T\left(e_{2}\right)(x)-x^{2} \leq x-x^{2}=$ $x(1-x)(0 \leq x \leq 1)$, where the operator $T$ is defined by (1.2). In particular we get $T\left(e_{2}\right)(0)=0$ and so Supp $\mu_{0}=\{0\}$; therefore $\mu_{0}=\varepsilon_{0}$. Moreover $T\left(e_{2}\right)(1)=1$, so $\int_{0}^{1}\left(e_{1}-e_{2}\right) d \mu_{1}=0$ and $\operatorname{Supp} \mu_{1} \subset\{0,1\}$. Then there exist $\alpha, \beta \in[0,1]$, $\alpha+\beta=1$, such that $\mu_{1}=\alpha \varepsilon_{0}+\beta \varepsilon_{1}$, so $1=\int_{0}^{1} e_{1} d \mu_{1}=\beta$. In conclusion we obtain $\alpha=0$ and $\mu_{1}=\varepsilon_{1}$. Finally we observe that, if $0 \leq x \leq 1$, then

$$
T\left(e_{2}\right)(x)=x^{2} \quad \text { if and only if } \mu_{x}=\varepsilon_{x} .
$$

Indeed, if $T\left(e_{2}\right)(x)=x^{2}$, considering the function $\psi_{x}(t)=(t-x)(0 \leq t \leq 1)$, we have $\int_{0}^{1} \psi_{x}^{2} d \mu_{x}=0$, so $\operatorname{Supp} \mu_{x}=\{x\}$ and $\mu_{x}=\varepsilon_{x}$. The converse is trivial.

From now on we suppose that the family $\left(\mu_{x}\right)_{1 \leq x \leq 1}$ satisfies the following further condition

$$
\mu_{x} \neq \varepsilon_{x} \quad \text { for every } 0<x<1 \text {. }
$$

Set

$$
\alpha(x):=\frac{1}{2}\left(T\left(e_{2}\right)(x)-x^{2}\right)=\frac{1}{2}\left(\int_{0}^{1} e_{2} d \mu_{x}-x^{2}\right) \quad(0 \leq x \leq 1) .
$$

Then $\alpha \in \mathcal{C}([0,1]), \alpha(0)=\alpha(1)=0$ and

$$
0<\alpha(x) \leq \frac{x(1-x)}{2} \text { for every } 0<x<1 .
$$

3.1. An asymptotic formula. Now consider the sequence $\left(B_{n}\right)_{n \geq 1}$ of the Bernstein-Schnabl operators associated with the continuous selection $\left(\mu_{x}\right)_{0 \leq x \leq 1}$. From definition (1.5) and from the above remarks it follows that, for every $n \geq 1$, $f \in \mathcal{C}([0,1])$,

$$
B_{n}(f)(x)=f(x) \quad \text { for } x=0,1 .
$$

In particular from (3.3) it follows that the operators $B_{n}$ map the space $\mathcal{C}_{0}(] 0,1[)$ into itself.

We now proceed to establish an asymptotic formula for the operators $B_{n}$.

Theorem 3.1. Consider the sequence $\left(B_{n}\right)_{n \geq 1}$ defined by (1.5). Then, for every $u \in \mathcal{C}^{2}([0,1])$,

$$
\lim _{n \rightarrow \infty} n\left(B_{n}(u)-u\right)=\alpha u^{\prime \prime} \quad \text { uniformly on }[0,1],
$$

where $\alpha$ is defined by (3.2). 
Proof. First note that from (1.6),(1.12) and (1.13) it follows that $\lim _{n \rightarrow \infty} n\left(B_{n}(\mathbf{1})\right.$ -1) $=\lim _{n \rightarrow \infty} n B_{n}\left(\psi_{x}\right)(x)=0$ and $\lim _{n \rightarrow \infty} n B_{n}\left(\psi_{x}^{2}\right)(x)=T\left(e_{2}\right)(x)-x^{2}$ uniformly with respect to $x \in[0,1]$. On the other hand, since $\psi_{x}^{4}=e_{4}-4 x e_{3}+$ $6 x^{2} e_{2}-4 x^{3} e_{1}+x^{4} \mathbf{1}(0 \leq x \leq 1)$, by using (1.6)-(1.10), we obtain

$$
\begin{aligned}
B_{n}\left(\psi_{x}^{4}\right)(x)= & \frac{1}{n^{3}}\left[T\left(e_{4}\right)(x)-4 x T\left(e_{3}\right)(x)+3(n-1) T\left(e_{2}\right)^{2}(x)\right. \\
& \left.-6(n-2) x^{2} T\left(e_{2}\right)(x)+3(n-2) x^{4}\right]
\end{aligned}
$$

and hence $\lim _{n \rightarrow \infty} n B_{n}\left(\psi_{x}^{4}\right)(x)=0$ uniformly with respect to $x \in[0,1]$. Accordingly the result follows from [18] (see also [5, Theorem 1]).

3.2. Degenerate differential operators on $[0,1]$. The asymptotic formula (3.4) naturally leads to the differential operator $Z(u)=\alpha u^{\prime \prime}\left(u \in \mathcal{C}^{2}([0,1])\right)$. In this subsection we shall investigate whether such differential operators are the pregenerators of Markov semigroups, i.e., they admit an extension which is the generator of a positive $\mathcal{C}_{0}$-semigroup $(T(t))_{t \geq 0}$ on $\mathcal{C}([0,1])$ such that $T(t) \mathbf{1}=\mathbf{1}$ for every $t \geq 0$. For more details about the theory of $\mathcal{C}_{0}$-semigroups we refer the reader to $[13,23]$.

We also recall that, if $(T(t))_{t \geq 0}$ is a $\mathcal{C}_{0}$-semigroup on $\mathcal{C}([0,1])$ with generator $(A, D(A))$, then $T(t) \mathbf{1}=\mathbf{1}$ for every $t \geq 0$ if and only if $\mathbf{1} \in D(A)$ and $A \mathbf{1}=0$.

Consider an arbitrary function $\alpha \in \mathcal{C}([0,1])$ such that $\alpha(0)=\alpha(1)=0$ and assume that $0<\alpha(x) \leq \frac{x(1-x)}{2}(0<x<1)$. If we set

$$
\lambda(x):=\frac{2 \alpha(x)}{x(1-x)} \quad(0<x<1)
$$

we have that $\lambda \in \mathcal{C}(] 0,1[), 0<\lambda(x) \leq 1(0<x<1)$ and

$$
\alpha(x)=\frac{x(1-x)}{2} \lambda(x) \quad(0<x<1) .
$$

Set

$$
D_{V}(A):=\left\{u \in \mathcal{C}([0,1]) \cap \mathcal{C}^{2}(] 0,1[) \mid \lim _{x \rightarrow 0^{+}} \alpha(x) u^{\prime \prime}(x)=\lim _{x \rightarrow 1^{-}} \alpha(x) u^{\prime \prime}(x)=0\right\}
$$

and

$$
D_{V}(B):=\left\{u \in \mathcal{C}([0,1]) \cap \mathcal{C}^{2}(] 0,1[) \mid \lim _{x \rightarrow 0^{+}} x(1-x) u^{\prime \prime}(x)=\lim _{x \rightarrow 1^{-}} x(1-x) u^{\prime \prime}(x)=0\right\},
$$

i.e., we assume Ventcel's conditions at the boundary points 0 and 1. Clearly $D_{V}(B) \subset D_{V}(A)$. For every $u \in D_{V}(A)$ (resp. $\left.u \in D_{V}(B)\right)$ and $x \in[0,1]$ define

$$
\begin{aligned}
& A u(x):= \begin{cases}\alpha(x) u^{\prime \prime}(x) & \text { if } 0<x<1, \\
0 & \text { if } x=0,1\end{cases} \\
& B u(x):= \begin{cases}\frac{x(1-x)}{2} u^{\prime \prime}(x) & \text { if } 0<x<1, \\
0 & \text { if } x=0,1 .\end{cases}
\end{aligned}
$$


It is well-known that $\left(B, D_{V}(B)\right)$ and $\left(A, D_{V}(A)\right)$ generate some Markov semigroups on $\mathcal{C}([0,1])$ and $\mathcal{C}^{2}([0,1])$ is a core for $\left(B, D_{V}(B)\right)$ (see $[8$, Theorem 6.2.6; Theorem 6.3.5]).

We recall that, given a linear operator $A: D(A) \longrightarrow E$ defined on a linear subspace $D(A)$ of a Banach space $E$, a core for $(A, D(A))$ is a subspace $D_{0}$ of $D(A)$ which is dense in $D(A)$ for the graph norm $\|u\|_{A}:=\|u\|+\|A u\|$ $(u \in D(A))$. Moreover, a Feller semigroup on $\mathcal{C}_{0}(] 0,1[)$ is a $\mathcal{C}_{0}$-semigroup of positive linear contractions on $\mathcal{C}_{0}(] 0,1[)$. Now let

$$
D\left(A_{0}\right):=D_{V}(A) \cap \mathcal{C}_{0}(] 0,1[) \quad \text { and } \quad D\left(B_{0}\right):=D_{V}(B) \cap \mathcal{C}_{0}(] 0,1[)
$$

and

$$
\begin{aligned}
& A_{0}:=A_{\left.\right|_{D\left(A_{0}\right)}}: D\left(A_{0}\right) \longrightarrow \mathcal{C}_{0}(] 0,1[), \\
& B_{0}:=B_{\left.\right|_{D\left(B_{0}\right)}}: D\left(B_{0}\right) \longrightarrow \mathcal{C}_{0}(] 0,1[) .
\end{aligned}
$$

Note that, from (3.6), $D\left(B_{0}\right) \subset D\left(A_{0}\right)$ and $A_{\left.0\right|_{D\left(B_{0}\right)}}=\lambda B_{0}$.

We have the following result.

Proposition 3.2. The operators $\left(B_{0}, D\left(B_{0}\right)\right)$ and $\left(A_{0}, D\left(A_{0}\right)\right)$ generate some Feller semigroups on $\mathcal{C}_{0}(] 0,1[)$. Moreover, $\mathcal{C}^{2}([0,1]) \cap \mathcal{C}_{0}(] 0,1[)$ is a core for $\left(B_{0}, D\left(B_{0}\right)\right)$.

Proof. The first part of the statement is a direct consequence of Proposition 2.2 in [7]. In order to prove the last part, fix $u \in D\left(B_{0}\right)$. Since $D\left(B_{0}\right) \subset D_{V}(B)$ and $\mathcal{C}^{2}([0,1])$ is a core for $\left(B, D_{V}(B)\right)$, there exists $\left(u_{n}\right)_{n \geq 1}$ in $\mathcal{C}^{2}([0,1])$ such that $u_{n} \rightarrow u$ and $B u_{n} \rightarrow B u$ uniformly on $[0,1]$ (the functions $B u_{n}$ and $B u$ are extended to 0 at the boundary points 0 and 1$)$. In particular $u_{n}(0) \rightarrow 0$ and $u_{n}(1) \rightarrow 0$. For every $n \geq 1$ set $v_{n}(x):=u_{n}(x)-x u_{n}(1)-(1-x) u_{n}(0)$. Then $v_{n} \in \mathcal{C}^{2}([0,1]) \cap \mathcal{C}_{0}(] 0,1[), v_{n} \rightarrow u$ uniformly on $[0,1]$ and finally $B v_{n}=$ $B u_{n} \longrightarrow B u$ uniformly on $[0,1]$.

In order to determine a core for the operator $A$ we consider the following subset of $D\left(A_{0}\right)$ :

$$
\begin{aligned}
D_{*}\left(A_{0}\right):=\left\{u \in D\left(A_{0}\right) \mid\right. & \text { There exists }\left(u_{n}\right)_{n \geq 1} \text { in } D\left(B_{0}\right) \text { such that } \\
& \left.u_{n} \rightarrow u \text { and } A_{0} u_{n} \rightarrow A_{0} u \text { uniformly on }[0,1]\right\} .
\end{aligned}
$$

Clearly $D\left(B_{0}\right) \subset D_{*}\left(A_{0}\right)$.

Theorem 3.3. The operator $\left(A_{0}, D_{*}\left(A_{0}\right)\right)$ is the generator of a Feller semigroup on $\mathcal{C}_{0}(] 0,1[)$ and $\mathcal{C}^{2}([0,1]) \cap \mathcal{C}_{0}(] 0,1[)$ is a core for $\left(A_{0}, D_{*}\left(A_{0}\right)\right)$. 
Proof. From Proposition 3.2 it follows that $\left(B_{0}, D\left(B_{0}\right)\right)$ is the generator of a Feller semigroup on $\mathcal{C}_{0}(] 0,1[)$. Thanks to [6, Corollary 2.7], $\left(\lambda B_{0}, D\left(B_{0}\right)\right)$ is closable and its closure $(C, D(C))$ generates a Feller semigroup on $\mathcal{C}_{0}(] 0,1[)$. Since $\left(A_{0}, D\left(A_{0}\right)\right)$ is closed, $D(C)=D_{*}\left(A_{0}\right)$ and $A_{0_{D_{*}\left(A_{0}\right)}}=C$. Finally, if $u \in D_{*}\left(A_{0}\right)$, fixed $\varepsilon>0$, there exists $v \in D\left(B_{0}\right)$ such that $\|u-v\| \leq \frac{\varepsilon}{2}$ and $\left\|A_{0} u-A_{0} v\right\| \leq \frac{\varepsilon}{2}$. On the other hand, by Proposition 3.2, there exists $w \in \mathcal{C}^{2}([0,1]) \cap \mathcal{C}_{0}(] 0,1[)$ such that $\|v-w\| \leq \frac{\varepsilon}{2}$ and $\left\|B_{0} v-B_{0} w\right\| \leq \frac{\varepsilon}{2}$. Then $\|u-w\| \leq\|u-v\|+\|v-w\| \leq \varepsilon$ and $\left\|A_{0} u-A_{0} w\right\| \leq\left\|A_{0} u-A_{0} v\right\|+\| \lambda B_{0} v-$ $\lambda B_{0} w \| \leq \varepsilon$.

We are now in a position to show the desired generation result on the space $\mathcal{C}([0,1])$. To this end, set

$$
\begin{aligned}
D_{V}^{*}(A):=\left\{u \in D_{V}(A) \mid\right. & \text { There exists }\left(u_{n}\right)_{n \geq 1} \text { in } D_{V}(B) \text { such that } \\
& \left.u_{n} \rightarrow u \text { and } A u_{n} \rightarrow A u \text { uniformly on }[0,1]\right\} .
\end{aligned}
$$

Obviously $D_{V}(B) \subset D_{V}^{*}(A)$. Moreover note that

$$
D_{V}^{*}(A) \cap \mathcal{C}_{0}(] 0,1[)=D_{*}\left(A_{0}\right) .
$$

Indeed, clearly $D_{*}\left(A_{0}\right) \subset D_{V}^{*}(A) \cap \mathcal{C}_{0}(] 0,1[)$. Conversely, fix $u \in D_{V}^{*}(A) \cap$ $\mathcal{C}_{0}(] 0,1[)$. Then $u \in D_{V}(A) \cap \mathcal{C}_{0}(] 0,1[)=D\left(A_{0}\right)$ and there exists a sequence $\left(u_{n}\right)_{n \geq 1}$ in $D_{V}(B)$ such that $u_{n} \rightarrow u$ and $A u_{n} \rightarrow A u$ uniformly on $[0,1]$. In particular $u_{n}(0) \rightarrow 0$ and $u_{n}(1) \rightarrow 1$. For every $n \geq 1$ set

$$
v_{n}(x):=u_{n}(x)-x u_{n}(1)-(1-x) u_{n}(0) \quad(0 \leq x \leq 1) .
$$

Then $v_{n} \in D\left(B_{0}\right), v_{n} \rightarrow u$ uniformly on $[0,1]$ and $A_{0} v_{n}=A v_{n} \rightarrow A u=A_{0} u$ uniformly on $[0,1]$. Therefore $u \in D_{*}\left(A_{0}\right)$.

Theorem 3.4. The operator $\left(A, D_{V}^{*}(A)\right)$ is the generator of a Markov semigroup $(T(t))_{t \geq 0}$ on $\mathcal{C}([0,1])$ and $\mathcal{C}^{2}([0,1])$ is a core for $\left(A, D_{V}^{*}(A)\right)$.

Proof. The first part of the statement will be proved by a generation result of Bony, Courrège, Priouret [10]. Indeed, $D_{V}^{*}(A)$ is dense in $\mathcal{C}([0,1])$, since $D_{V}(B)$ is dense in $\mathcal{C}([0,1])$. Moreover, the operator $\left(A, D_{V}^{*}(A)\right)$ satisfies the positive maximum principle (see $[10])$, since $\left(A, D_{V}(A)\right)$ satisfies it.

Finally we proceed to show that $R(\lambda I-A)=\mathcal{C}([0,1])$ for $\lambda>0$ fixed. Let $f \in \mathcal{C}([0,1])$ and consider the function $g(x):=f(x)-x f(1)-(1-x) f(0)$. Then $g \in \mathcal{C}_{0}(] 0,1[)$. From Theorem 3.3, $\left(\lambda I-A_{0}\right)\left(D_{*}\left(A_{0}\right)\right)=\mathcal{C}_{0}(] 0,1[)$ and so there exists an element $u \in D_{*}\left(A_{0}\right)$ such that $\lambda u-A_{0} u=g$. Now set

$$
v(x):=u(x)+\frac{1}{\lambda}[x f(1)+(1-x) f(0)] \quad(0 \leq x \leq 1)
$$


(the function $u$ is continuously extended at 0 and 1 ). Since $u \in D\left(A_{0}\right) \subset D_{V}(A)$, $v \in D_{V}(A)$ and $A v=A_{0} u$ on $[0,1]$. Moreover $\lambda v-A v=f$.

In order to show the last part, let $u \in D_{V}^{*}(A)$ and $\varepsilon>0$ and consider the function $\lambda \in \mathcal{C}_{b}(] 0,1[)$ defined by (3.5), so that $\alpha(x)=\frac{x(1-x)}{2} \lambda(x)(0 \leq x \leq 1)$. Take $v \in D_{V}(B)$ such that $\|u-v\| \leq \frac{\varepsilon}{2}$ and $\|A u-A v\| \leq \frac{\varepsilon}{2}$, and let $w \in \mathcal{C}^{2}([0,1])$ such that $\|v-w\| \leq \frac{\varepsilon}{2}$ and $\|B v-B w\| \leq \frac{\varepsilon}{2}$. Then $\|u-w\| \leq\|u-v\|+\|v-w\| \leq$ $\varepsilon$. Moreover if $x=0,1$, then $|A u(x)-A w(x)|=0 \leq \varepsilon$ and, if $0<x<1$,

$$
\begin{aligned}
|A u(x)-A w(x)| & \leq|A u(x)-A v(x)|+|A v(x)-A w(x)| \\
& \leq \frac{\varepsilon}{2}+|\lambda(x) B v(x)-\lambda(x) B w(x)| \\
& \leq \frac{\varepsilon}{2}+|B v(x)-B w(x)| \leq \varepsilon .
\end{aligned}
$$

Accordingly $\|A u-A w\| \leq \varepsilon$.

3.3. Markov semigroups associated with Bernstein-Schnabl operators. We turn back to the Bernstein-Schnabl operators associated with a continuous selection of probability Borel measures $\left(\mu_{x}\right)_{0 \leq x \leq 1}$ on $[0,1]$ which satisfies (1.3) and (3.1).

For every $p \geq 1$ the power $B_{n}^{p}$ of order $p$ of the operator $B_{n}$ is defined by

$$
B_{n}^{p}:= \begin{cases}B_{n} & p=1 \\ B_{n} \circ B_{n}^{p-1} & p \geq 2 .\end{cases}
$$

Theorem 3.5. Let $\alpha$ be the function defined by (3.2) and consider the operators $\left(A_{0}, D_{*}\left(A_{0}\right)\right)$ and $\left(A, D_{V}^{*}(A)\right)$ defined by (3.10), (3.11) and (3.8), (3.12), respectively. Then these operators generate a Feller semigroup $(S(t))_{t \geq 0}$ and a Markov semigroup $(T(t))_{t \geq 0}$ on $\mathcal{C}_{0}(] 0,1[)$ and $\mathcal{C}([0,1])$, respectively. Moreover, for every $t \geq 0, S(t)=T(t)_{\left.\right|_{\left.\mathcal{C}_{0}(] 0,1\right]}}$ and, for every sequence $(k(n))_{n \geq 1}$ of positive integers such that $\frac{k(n)}{n} \rightarrow t$ and for every $f \in \mathcal{C}([0,1])$,

$$
T(t) f=\lim _{n \rightarrow \infty} B_{n}^{k(n)} f \quad \text { uniformly on }[0,1] .
$$

Proof. The first part of the statement follows from Theorems 3.3 and 3.4. Moreover, by Theorem 3.1, for every $u \in \mathcal{C}^{2}([0,1]) \subset D_{V}^{*}(A)$

$$
\lim _{n \rightarrow \infty} n\left(B_{n}(u)-u\right)=A u \quad \text { uniformly on }[0,1]
$$

and $\mathcal{C}^{2}([0,1])$ is a core for $\left(A, D_{V}^{*}(A)\right)$. Finally, for every $n \geq 1$ and $p \geq 1$, $\left\|B_{n}^{p}\right\|=1$ since $B_{n}^{p}(\mathbf{1})=\mathbf{1}$. Therefore, from Trotter's theorem (see [13, Corollary 5.8]; see also [8, Theorem 1.6.7]) formula (3.14) easily follows. 
Finally, fix $u_{0} \in D_{*}\left(A_{0}\right) \subset D_{V}^{*}(A)$ and consider the function $u(t):=T(t) u_{0}$ $(t \geq 0)$. Then $u(t) \in D_{V}^{*}(A)$ for every $t \geq 0, u$ is strongly differentiable in $\left[0,+\infty\left[\right.\right.$ and $\frac{d u(t)}{d t}=A u(t)$ for every $t \geq 0$. From (3.14), (3.4) and (3.13), it follows that $T(t) u_{0} \in D_{V}^{*}(A) \cap \mathcal{C}_{0}(] 0,1[)=D_{*}\left(A_{0}\right)$ and so, by the uniqueness of the solution of the Cauchy problem associated with $\left(A_{0}, D_{*}\left(A_{0}\right)\right)$, we get that $u(t)=S(t) u_{0}$ for every $t \geq 0$. Therefore $T(t)=S(t)$ on $D_{*}\left(A_{0}\right)$ and hence on $\mathcal{C}_{0}(] 0,1[)$, because $D_{*}\left(A_{0}\right)$ is dense in $\mathcal{C}_{0}(] 0,1[)$.

From Theorem 3.5 and the main result of Section 2 the following Corollary immediately follows. It collects some qualitative properties of the semigroups indicated in Theorem 3.5, and hence of the solutions of the Cauchy problems associated with $\left(A, D_{V}^{*}(A)\right)$ (or with $\left(A_{0}, D_{*}\left(A_{0}\right)\right)$ ) which are given by $u(t)=T(t) u_{0}, u_{0} \in D_{V}^{*}(A)$ (resp., $\left.u(t)=S(t) u_{0}, u_{0} \in D_{*}\left(A_{0}\right)\right)$.

Corollary 3.6. Under the same assumptions of Theorem 3.5, the following statements hold true:

(1) If the operator $T$ given by (1.2) maps continuous increasing functions into (continuous) increasing functions, then each $T(t)$ maps continuous increasing functions into increasing functions.

(2) If $T\left(\right.$ Lip $\left._{1} 1\right) \subset$ Lip $_{1} 1$, then for every $M>0,0<\alpha \leq 1$ and $t \geq 0$,

$$
T(t)\left(\operatorname{Lip}_{M} \alpha\right) \subset \operatorname{Lip}_{M} \alpha .
$$

(3) If $f \in \mathcal{C}([0,1])$ the following statements are equivalent:

(i) $f$ is convex;

(ii) $f \leq T(t) f$ for every $t \geq 0$.

(4) Under the assumptions $\left(c_{1}\right)$ and $\left(c_{2}\right)$ of Section 2 , if $f \in \mathcal{C}([0,1])$ is convex, then each $T(t) f$ is convex $(t \geq 0)$ and $(T(t) f)_{t \geq 0}$ is increasing.

Proof. Statements (1) and (2) follow from Theorem 2.1 and Corollary 2.4. The implication $(i) \Rightarrow(i i)$ follows from (2.4). Conversely, assume that $f \leq T(t) f$ for every $t \geq 0$ and set $u(r):=\frac{1}{r} \int_{0}^{r} T(s) f d s \in D_{V}^{*}(A)$ for every $r>0$. Then $A u(r)=\frac{1}{r}[T(r) f-f] \geq 0$, so that $u(r)$ is convex for every $r>0$. Accordingly $f=\lim _{r \rightarrow 0^{+}} u(r)$ is convex too.

The first part of (4) is a consequence of Theorem 2.6. As regard to the second part, we first consider $u \in D_{V}^{*}(A), u$ convex. Then for every $t \geq 0$, $T(t) \in D_{V}^{*}(A)$ and $T(t) u$ is convex. Therefore $A T(t) u \geq 0$ and hence $\frac{d}{d t} T(t) u=$ $A T(t) u \geq 0 \quad(t \geq 0)$, so that $(T(t) u)_{t \geq 0}$ is increasing. We now consider an arbitrary convex function $f \in \mathcal{C}([0,1])$. Set $u(r):=\frac{1}{r} \int_{0}^{r} T(s) f d s \in D_{V}^{*}(A)$ for any $r>0$. Then $\lim _{r \rightarrow 0^{+}} u(r)=f$ uniformly on $[0,1]$ and each $u(r)$ is convex as we have previously proved. So, for $0 \leq s<t$ we get $T(s) u(r) \leq T(t) u(r)$ and hence $T(s) f \leq T(t) f$ because of the continuity of the operators $T(s)$ and $T(t)$ and this finishes the proof. 
In order to investigate the long-time behaviour of the semigroup $(T(t))_{t \geq 0}$ we shall use the next result which generalizes Theorem 2 of [17].

Let $(E,\|\cdot\|)$ be a Banach space of real-valued functions defined on a convex subset $X$ of a locally convex space. Assume that the space $E$, endowed with its norm and the pointwise order, is a Banach lattice.

Proposition 3.7. Let $\left(L_{i}\right)_{i \in I}^{\leq}$be a net of positive linear operators from $E$ into itself and assume that for every convex function $\varphi \in E$, the net $\left(L_{i}(\varphi)\right)_{i \in I}^{\leq}$ is decreasing (resp., increasing). Further assume that for some convex function $u \in E$, the net $\left(L_{i}(u)\right)_{i \in I}^{\leq}$is convergent in $E$. Then, for every $f \in$ $\mathcal{A}(u):=\{g \in E \mid$ there exists $\lambda \geq 0$ such that $\lambda u-g$ and $\lambda u+g$ are convex $\}$ the net $\left(L_{i}(f)\right)_{i \in I}^{\leq}$is convergent in $E$. Therefore, if $\mathcal{A}(u)$ is dense in $E$ and $\sup _{i \in I, i_{0} \leq i}\left\|L_{i}\right\|<+\infty$ for some $i_{0} \in I$, then $\left(L_{i}(f)\right)_{i \in I}^{\leq}$is convergent in $E$ for every $f \in E$.

Proof. Consider $f \in E$ and assume that $\lambda u-f$ and $\lambda u+f$ are convex for some $\lambda \geq 0$. Since $E$ is a Banach space, in order to show that the net $\left(L_{i}(f)\right)_{i \in I}^{\leq}$is convergent it is enough to prove that it is a Cauchy net (see [19, Definition 2.1.41 and Corollary 2.1.51]). To this end, first observe that, for every $i, j \in I, i \leq j$, we have $L_{j}(\lambda u-f) \leq L_{i}(\lambda u-f)$ and $L_{j}(\lambda u+f) \leq L_{i}(\lambda u+f)$, so that $L_{i}(f)-L_{j}(f) \leq \lambda\left(L_{i}(u)-L_{j}(u)\right)$ and $L_{j}(f)-L_{i}(f) \leq \lambda\left(L_{i}(u)-L_{j}(u)\right)$. Hence

$$
\left|L_{i}(f)-L_{j}(f)\right| \leq \lambda\left|L_{i}(u)-L_{j}(u)\right|
$$

and so

$$
\left\|L_{i}(f)-L_{j}(f)\right\| \leq \lambda\left\|L_{i}(u)-L_{j}(u)\right\| .
$$

For arbitrary $i, j \in I$, chosen $k \in I$ such that $i \leq k$ and $j \leq k$, we have

$$
\left\|L_{i}(f)-L_{j}(f)\right\| \leq \lambda\left(\left\|L_{i}(u)-L_{k}(u)\right\|+\left\|L_{j}(u)-L_{k}(u)\right\|\right) .
$$

From the above inequality it follows that $\left(L_{i}(f)\right)_{i \in I}^{\leq}$is a Cauchy net because so is the net $\left(L_{i}(u)\right)_{i \in I}^{\leq}$.

\section{Remarks 3.8.}

1. We point out that Proposition 3.7 remains valid by replacing everywhere the class of convex functions on $X$ with an arbitrary class of real-valued functions on an arbitrary set $X$.

2. Under the hypotheses of the last part of Proposition 3.7, we can consider the positive linear operator $L_{0}: E \longrightarrow E$ defined by

$$
L_{0}(f):=\lim _{i \in I} L_{i}(f) \quad(f \in E)
$$


For fixed $f \in \mathcal{A}(u)$ and $i \in I$, the net $\left(L_{j}(f)\right)_{j \in I, i \leq j}^{\leq}$is a subnet of $\left(L_{k}(f)\right)_{k \in I}^{\leq}$ and, therefore, it converges to $L_{0}(f)$. From (3.16) it follows that

$$
\left\|L_{i}(f)-L_{0}(f)\right\| \leq \lambda\left\|L_{i}(u)-L_{0}(u)\right\|
$$

for every $i \in I$ and $f \in \mathcal{A}(u)$. If, in addition, the convergence in norm in $E$ implies the pointwise convergence, then from (3.15) we also get

$$
\left|L_{i}(f)-L_{0}(f)\right| \leq \lambda\left|L_{i}(u)-L_{0}(u)\right|
$$

3. If $X$ is a real interval and the convex function $u$ belongs to $\mathcal{C}^{2}(X)$, then $\left\{f \in E \cap \mathcal{C}^{2}(X)|| f^{\prime \prime} \mid \leq \lambda u^{\prime \prime}\right.$ for some $\left.\lambda \geq 0\right\} \subset \mathcal{A}(u)$. In particular, if $\alpha:=$ $\min _{X} u^{\prime \prime}(x)>0$, then $\left\{f \in E \cap \mathcal{C}^{2}(X) \mid f^{\prime \prime}\right.$ bounded $\} \subset \mathcal{A}(u)$, since $\left|f^{\prime \prime}\right| \leq \frac{\left\|f^{\prime \prime}\right\|}{\alpha} u^{\prime \prime}$ for every $f \in E \cap \mathcal{C}^{2}(X), f^{\prime \prime}$ bounded.

We can now state a result about the asymptotic behaviour of the semigroup $(T(t))_{t \geq 0}$.

Theorem 3.9. Under the same assumptions of Theorem 3.5, suppose that $T\left(\right.$ Lip $\left._{1} 1\right) \subset$ Lip $_{1} 1$ and both conditions $\left(c_{1}\right)$ and $\left(c_{2}\right)$ are satisfied. Then there exists a positive linear operator $T_{\infty}: \mathcal{C}([0,1]) \longrightarrow \mathcal{C}([0,1])$ such that

$$
\lim _{t \rightarrow+\infty} T(t) f=T_{\infty}(f) \quad \text { uniformly on }[0,1]
$$

for every $f \in \mathcal{C}([0,1])$. Moreover $T_{\infty}(\mathbf{1})=\mathbf{1}$ and, for every $f \in \mathcal{C}^{2}([0,1])$ and $t \geq 0$,

$$
\left|T(t) f-T_{\infty}(f)\right| \leq \frac{\left\|f^{\prime \prime}\right\|_{\infty}}{2}\left|T(t) e_{2}-T_{\infty}\left(e_{2}\right)\right| .
$$

Finally, for every $f \in \mathcal{C}([0,1]), x \in[0,1]$ and $t \geq 0$,

$$
\left|T(t) f(x)-T_{\infty}(f)(x)\right| \leq M \omega_{2}\left(f, \sqrt{\lambda_{t}(x)}\right),
$$

where $\lambda_{t}(x)=\left|T(t) e_{2}(x)-T_{\infty}\left(e_{2}\right)(x)\right|$ and $M$ is an absolute constant.

Proof. We shall use Proposition 3.7 with $E=\mathcal{C}([0,1])$, endowed with the uniform norm, and $u:=e_{2}$. In this case $\mathcal{C}^{2}([0,1]) \subset \mathcal{A}(u)$ and $\sup _{t \geq 0}\|T(t)\|=1$. Therefore, taking Corollary 3.6, (3), into account, it is sufficient to show that $\left(T(t) e_{2}\right)_{t \geq 0}$ is uniformly convergent on $[0,1]$ as $t \rightarrow+\infty$. Actually this family is increasing and bounded so that it is pointwise convergent. Since $e_{2} \in \operatorname{Lip}_{2} 1$, by Corollary 3.6, (2), $T(t) e_{2} \in \operatorname{Lip}_{2} 1$ for every $t \geq 0$. Therefore $\left(T(t) e_{2}\right)_{t \geq 0}$ is equicontinuous and hence uniformly convergent on $[0,1]$. Finally, the estimate (3.19) follows from (3.17). To obtain (3.20), let us consider the Peetre K-functional

$$
K_{2}(f, \delta):=\inf \left\{\|f-g\|_{\infty}+\delta\left\|g^{\prime \prime}\right\|_{\infty}: g \in \mathcal{C}^{2}([0,1])\right\}
$$


$(f \in \mathcal{C}([0,1]), \delta>0)$ which is equivalent to $\omega_{2}(f, \cdot)$, i.e.,

$$
C_{1} \omega_{2}(f, \delta) \leq K_{2}\left(f, \delta^{2}\right) \leq C_{2} \omega_{2}(f, \delta)
$$

$(f \in \mathcal{C}([0,1]), \delta>0)$ with $C_{1}, C_{2}$ absolute constants (see, e.g., [11, Theorem 2.4, p. 177]). Given $f \in \mathcal{C}([0,1]), t \geq 0, x \in[0,1]$ and $\varepsilon>0$, there exists a $g \in \mathcal{C}^{2}([0,1])$ such that $\|f-g\|_{\infty}+\delta_{t}^{2}(x)\left\|g^{\prime \prime}\right\|_{\infty} \leq C_{2} \omega_{2}\left(f, \delta_{t}(x)\right)+\varepsilon$, where $\delta_{t}(x):=\sqrt{\lambda_{t}(x)} \geq 0$. Therefore

$$
\begin{aligned}
\left|T(t) f(x)-T_{\infty}(f)(x)\right| \leq & |T(t) f(x)-T(t) g(x)| \\
& +\left|T(t) g(x)-T_{\infty}(g)(x)\right|+\left|T_{\infty}(g)(x)-T_{\infty}(f)(x)\right| \\
\leq & \|T(t)\|\|f-g\|_{\infty}+\frac{\left\|g^{\prime \prime}\right\|_{\infty}}{2} \delta_{t}^{2}(x)+\left\|T_{\infty}\right\|\|f-g\|_{\infty} \\
\leq & 2\|f-g\|_{\infty}+\frac{\left\|g^{\prime \prime}\right\|_{\infty}}{2} \delta_{t}^{2}(x) \\
\leq & 2\left(C_{2} \omega_{2}\left(f, \delta_{t}(x)\right)+\varepsilon\right) .
\end{aligned}
$$

Letting $\varepsilon \rightarrow 0^{+}$we obtain (3.20) with $M=2 C_{2}$.

In the last part of the paper we actually show that the Markov semigroup generated by an arbitrary differential operator of the form (3.8) can be represented by iterates of Bernstein-Schnabl operators associated with a suitable selection of probability Borel measures on $[0,1]$.

Consider indeed an arbitrary function $\alpha \in \mathcal{C}([0,1])$ such that $\alpha(0)=\alpha(1)=0$ and $0<\alpha(x) \leq \frac{x(1-x)}{2}(0<x<1)$. Consider the operator $\left(A, D_{V}^{*}(A)\right)$, defined by (3.7) and (3.9). By Theorem 3.4 such an operator generates a Markov semigroup $(T(t))_{t \geq 0}$ on $\mathcal{C}([0,1])$.

One may ask whether there exists a continuous selection $\left(\mu_{x}\right)_{0 \leq x \leq 1}$ of probability Borel measures satisfying (1.3) and (3.1), such that the corresponding Bernstein-Schnabl operators represent the semigroup by means of their iterates, as in Theorem 3.5. To this respect we have the following result.

Theorem 3.10. Under the above hypotheses set $\lambda(x):=\frac{2 \alpha(x)}{x(1-x)}(0<x<1)$ and, for every $x \in[0,1]$, consider the measure

$$
\mu_{x}:= \begin{cases}\lambda(x)\left[x \varepsilon_{1}+(1-x) \varepsilon_{0}\right]+(1-\lambda(x)) \varepsilon_{x} & \text { if } 0<x<1 \\ \varepsilon_{x} & \text { if } x=0,1 .\end{cases}
$$

Let $\left(B_{n}\right)_{n \geq 1}$ the sequence of the Bernstein-Schnabl operators associated with the selection $\left(\mu_{x}\right)_{0 \leq x \leq 1}$ (see Examples $\left.1.2,2\right)$. Then for every $t \geq 0$, for each sequence $(k(n))_{n \geq 1}$ of natural integers such that $\frac{k(n)}{n} \rightarrow t(n \rightarrow \infty)$ and for every function $f \in \mathcal{C}([0,1])$

$$
T(t) f=\lim _{n \rightarrow \infty} B_{n}^{k(n)}(f) \quad \text { uniformly on }[0,1] .
$$


Proof. We preliminary observe that $\lambda \in \mathcal{C}_{b}(] 0,1[), 0<\lambda(x) \leq 1$ and $\alpha(x)=$ $\frac{x(1-x)}{2} \lambda(x)(0 \leq x \leq 1)$. The family $\left(\mu_{x}\right)_{0 \leq x \leq 1}$ is a continuous selection of probability Borel measures satisfying (1.3) and (3.1). Moreover $\int_{0}^{1} e_{2} d \mu_{x}=$ $x^{2}+x(1-x) \lambda(x)=x^{2}+2 \alpha(x)$. According to Theorem 3.5 the semigroup $(T(t))_{t \geq 0}$ is represented as the limit of iterates of the operators $B_{n}$ corresponding to the selection (3.21) and the proof is complete.

We conclude this paper with some remarks.

\section{Remarks 3.11.}

1. Assume that the function $\alpha$ is differentiable at 0 and 1 and $\alpha^{\prime}(0) \neq 0 \neq \alpha^{\prime}(1)$, that is, $T\left(e_{2}\right)$ is differentiable at 0 and 1 and $T\left(e_{2}\right)^{\prime}(0) \neq 0$ and $T\left(e_{2}\right)^{\prime}(1) \neq 2$. Then, the function $\lambda$ defined by (3.5) can be continuously extended at the points 0 and 1 and such extension, denoted again by $\lambda$, is strictly positive on $[0,1]$ and $\alpha(x)=\frac{x(1-x)}{2} \lambda(x)(0 \leq x \leq 1)$. In this case $D_{V}(B)=D_{V}(A)$, and hence $D_{V}^{*}(A)=D_{V}(A)$, and Theorem 3.10 generalizes a previous result obtained in [4] for the sequence of the Lototsky-Schnabl operators. Moreover, in [8, Theorem 6.2.6] it is also shown that (not necessarily under assumptions $\left(c_{1}\right)$ and $\left.\left(c_{2}\right)\right)$ the semigroup $(T(t))_{t \geq 0}$ strongly converges as $t \rightarrow+\infty$ to the positive operator

$$
T_{\infty}(f)=\left(1-e_{1}\right) f(0)+e_{1} f(1) \quad(f \in \mathcal{C}([0,1])) .
$$

It would be interesting to prove or disprove that the limit operator $T_{\infty}$ defined by (3.18) actually coincides with the operator (3.22).

2 . Let $\left(B_{n}\right)_{n \geq 1}$ be the sequence of the Bernstein-Schnabl operators associated with a continuous selection of measures $\left(\mu_{x}\right)_{0 \leq x \leq 1}$. Let $\alpha(x)=\frac{1}{2}\left(\int_{0}^{1} e_{2} d \mu_{x}-\right.$ $\left.x^{2}\right)(0 \leq x \leq 1)$ and consider the function $\lambda \in \mathcal{C}_{b}(] 0,1[)$ defined by (3.5). Moreover, consider the selection defined by (3.21) and the sequence $\left(L_{n, \lambda}\right)_{n \geq 1}$ of the relevant Bernstein-Schnabl operators associated with this selection, that is, the Lototsky-Schnabl operators associated with $\lambda$. Then, for every $u \in \mathcal{C}^{2}([0,1])$

$$
\lim _{n \rightarrow \infty} n\left(B_{n}(u)-u\right)=\alpha u^{\prime \prime}=\lim _{n \rightarrow \infty} n\left(L_{n, \lambda}(u)-u\right)
$$

uniformly on $[0,1]$, therefore

$$
\lim _{n \rightarrow \infty} n\left(B_{n}(u)-L_{n, \lambda}(u)\right)=0 \quad \text { uniformly on }[0,1] .
$$

In particular

$$
\lim _{n \rightarrow \infty} B_{n}(u)-L_{n, \lambda}(u)=0 \quad \text { uniformly on }[0,1] .
$$

Since $\sup _{n \geq 1}\left\|B_{n}\right\| \leq 1$ and $\sup _{n \geq 1}\left\|L_{n, \lambda}\right\| \leq 1$ and $\mathcal{C}^{2}([0,1])$ is dense in $\mathcal{C}([0,1])$, for every $f \in \mathcal{C}([0,1])$

$$
\lim _{n \rightarrow \infty} B_{n}(f)-L_{n, \lambda}(f)=0 \quad \text { uniformly on }[0,1] .
$$


Accordingly, for every $f \in \mathcal{C}([0,1])$ there exists $\left(\varphi_{n}\right)_{n \geq 1}$ in $\mathcal{C}([0,1])$ such that

$$
B_{n}(f)=L_{n, \lambda}(f)+\varphi_{n} \text { and }\left\|\varphi_{n}\right\| \rightarrow 0(n \rightarrow \infty)
$$

Moreover the semigroup represented by $\left(B_{n}\right)_{n \geq 1}$ is the same one represented by $\left(L_{n, \lambda}\right)_{n \geq 1}$.

These remarks could be useful to study the saturation class of the sequence $\left(B_{n}\right)_{n \geq 1}$.

Note added in proof. Under the general assumption of Section 3, as a consequence of Theorem 3.4, it is possible to show that $C^{2}([0,1])$ is a core for $\left(A, D_{V}(A)\right)$ and hence $D_{V}^{*}(A)=D_{V}(A)$.

Choosing indeed $\lambda>0$, then $(\lambda I-A)\left(C^{2}([0,1])\right)$ is dense in $C([0,1])$ because $C^{2}([0,1])$ is a core for $\left(A, D_{V}^{*}(A)\right)$. Since $\left(A, D_{V}(A)\right)$ generates a Feller semigroup too, the above density relation implies in turn that $C^{2}([0,1])$ is a core for $\left(A, D_{V}(A)\right)$ and hence the two domains coincide.

We thank Sabina Milella for pointing out this useful remark.

Acknowledgement. The first author acknowledges the support of the University of Bari. The second author also acknowledges the support of the "Istituto Per Ricerche ed Attività Educative" of Napoli.

\section{References}

[1] Adell, J. A., de la Cal, J. and Raşa, I., Lototsky-Schnabl operators on the unit interval. Rend. Circ. Mat. Palermo (2) 48 (1999)(3), 517 - 536.

[2] Altomare, F., Limit semigroups of Bernstein-Schnabl operators associated with positive projections. Ann. Sc. Norm. Sup. Pisa, Cl. Sci. (4) 16 (1989)(2), $259-279$.

[3] Altomare, F., Positive projections, approximation processes and degenerate diffusion equations. In: Recent developments in mathematical analysis and its applications (Bari, Italy, 1990). Conf. Sem. Mat. Univ. Bari 241 (1991), $43-68$.

[4] Altomare, F., Lototsky-Schnabl operators on the unit interval and degenerate diffusion equations. In: Progress in Functional Analysis (Peñiscola, Spain, 1990; eds.: K. D. Bierstedt et al.). North-Holland Math. Studies 170. Amsterdam: North-Holland 1992, pp. $259-277$.

[5] Altomare, F. and Amiar, R., Asymptotic formulae for positive linear operators. Math. Balkanica (NS) 16 (2002), 283 - 304.

[6] Altomare, F. and Attalienti, A., Degenerate evolution equations in weighted continuous function spaces, Feller process and the Black-Scholes equation, Part I. Result. Math. 42 (2002), $193-211$. 
[7] Altomare, F. and Attalienti, A., Degenerate evolution equations in weighted continuous function spaces, Feller process and the Black-Scholes equation, Part II. Result. Math. 42 (2002), 212 - 228.

[8] Altomare, F. and Campiti, M., Korovkin-Type Approximation Theory and its Applications. De Gruyter Studies Math. 17. Berlin: de Gruyter 1994.

[9] Bauer, H., Probability Theory. De Gruyter Studies Math. 23. Berlin: de Gruyter 1996.

[10] Bony, J.-M., Courège, P. and Priouret, P., Semi-groupes de Feller sur une variété à bord compacte et problèmes aux limites intégro-différentiels du second ordre donnant lieu au principe de maximum. Ann. Inst. Fourier (Grenoble) 18 (1968), $369-521$.

[11] DeVore, R. A. and Lorentz, G. G., Constructive Approximation. Grundlehren der Math. Wissenschaften 303. Berlin: Springer 1993.

[12] Ditzian, Z. and Totik, V., Moduli of Smothness. Springer Ser. Comput. Math. 9. New York: Springer 1987.

[13] Engel, K.-J. and Nagel, R., One-Parameter Semigroups for Linear Evolution Equations. Grad. Texts Math. 194. New York: Springer 2000.

[14] Gavrea, I., Gonska, H., Păltănea, R. and Tachev, G., General estimates for the Ditzian-Totik modulus. East J. Approx. 9 (2003)(2), 175 - 194.

[15] Gonska, H., Positive operators and approximation of functions: selected topics. Conf. Sem. Mat. Univ. Bari 288 (2002).

[16] Grossman, M. W., Note on a generalized Bohman-Korovkin theorem. J. Math. Anal. Appl. 45 (1974), 43 - 46.

[17] Heping, W., Korovkin-type theorem and application. J. Approx. Theory 132 (2005)(2), $258-264$.

[18] Mamedov, R. G., On the order of the approximation of differentiable functions by linear positive operators (in Russian). Dokl. Akad. Nauk SSSR 146 (1962), 1013 - 1016; translated in: Soviet Mathematics 3 (1962)(5), 1435 - 1439.

[19] Megginson, R. E., An Introduction to Banach Space Theory. Grad. Texts Math. 183. New York: Springer 1998.

[20] Nishishiraho, T., Quantitative theorems on approximations processes of positive linear operators. In: Multivariate Approximation Theory II (Oberwolfach 1982; eds.: W. Schempp et al.). Internat. Ser. Numer. Math. 61. Basel: Birkhäuser 1982, pp. $297-311$.

[21] Nishishiraho, T., The order of approximation by positive linear operators. Tôhoku Math. J. 40 (1988)(4), 617 - 632.

[22] Păltănea, R., Approximation Theory Using Positive Linear Operators. Boston (MA): Birkhäuser 2004.

[23] Pazy, A., Semigroups of Linear Operators and Applications to Partial Differential Equations. Berlin: Springer 1983. 
[24] Raşa, I., Generalized Bernstein operators and convex functions. Studia Univ. Babeş-Bolyai Math. 33 (1988)(2), 36 - 39.

[25] Raşa, I., On the monotonicity of sequences of Bernstein-Schnabl operators. Anal. Numér. Théor. Approx. 17 (1988)(2), 185 - 187.

[26] Raşa, I., Altomare projections and Lototsky-Schnabl operators. Proc. 2nd Intern. Confer. Functional Analysis and Approximation Theory (Acquafredda di Maratea, Italy, 1992). Rend. Circ. Mat. Palermo (2) Suppl. 33 (1993), $439-451$.

[27] Schnabl, R., Eine Verallgemeinerung der Bernsteinpolynome. Math. Ann. 179 (1968), $74-82$.

[28] Schnabl, R., Zur Approximation durch Bernstein Polynome auf gewissen Räumen von Wahrscheinlichkeitsmaßen. Math. Ann. 180 (1969), 326 - 330.

[29] Sendov, B. and Popov, V. A., The Averaged Moduli of Smoothness. Pure Appl. Mathematics. Chichester: Wiley 1988.

Received March 16, 2006 